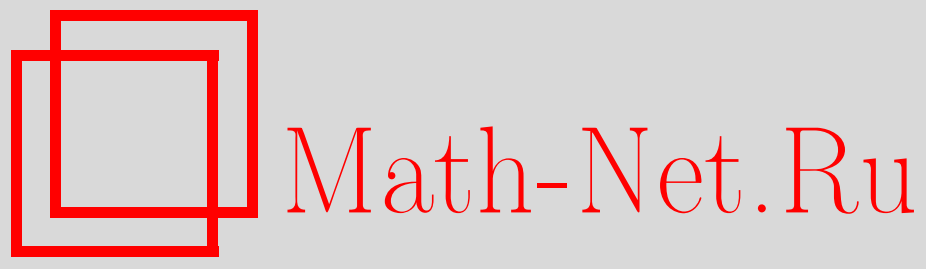

В. А. Быковский, Относительные минимумы решеток и вершины многогранников Клейна, Функи. анализ и его прил., 2006, том 40, выпуск 1, 69-71

DOI: https://doi.org/10.4213/faa20

Использование Общероссийского математического портала Math-Net.Ru подразумевает, что вы прочитали и согласны с пользовательским соглашением

http://www . mathnet.ru/rus/agreement

Параметры загрузки:

IP: 54.174 .149 .18

26 апреля 2023 г., 12:07:33

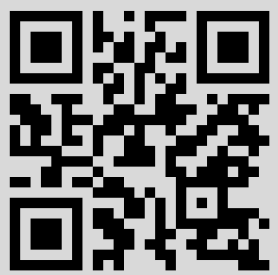




\title{
Относительные минимумы решеток и вершины многогранников Клейна*
}

\author{
(c) 2006. В. А. Быковский
}

В конце девятнадцатого века были предложены два многомерных обобщения классического алгоритма цепных дробей: одно - Ф. Клейном [1], а второе Г. Ф. Вороным [2] и независимо Г. Минковским [3]. В каждом из них среди узлов $s$-мерной решетки

$$
\Gamma=\left\{m_{1} \gamma^{(1)}+\cdots+m_{s} \gamma^{(s)} \mid m_{1}, \ldots, m_{s} \in \mathbb{Z}\right\},
$$

с базисными узлами $\gamma^{(1)}, \ldots, \gamma^{(s)}$ из $\mathbb{R}^{s}$ выделяется и изучается специальное подмножество узлов, $\mathfrak{N}(\Gamma)$ и $\mathfrak{M}(\Gamma)$ соответственно.

В конструкции Клейна $\mathfrak{N}(\Gamma)$ состоит из всех вершин многогранников Клейна $\mathscr{K}_{\theta}(\Gamma), \theta=\left(\theta_{1}, \ldots, \theta_{s}\right), \theta_{i}= \pm 1$. Напомним, что $\mathscr{K}_{\theta}(\Gamma)$ определяется как выпуклая оболочка ненулевых узлов решетки $\Gamma$, содержащихся в $s$-гранном угле

$$
\left\{\left(\theta_{1} t_{1}, \ldots, \theta_{s} t_{s}\right) \in \mathbb{R}^{s} \mid t_{i} \in[0, \infty), i=1, \ldots, s\right\} .
$$

Недавно вышедшая популярная брошюра В. И. Арнольда [4] содержит много интересных фактов по поводу этой конструкции.

В конструкции Вороного и Минковского $\mathfrak{M}(\Gamma)$ состоит из всех ненулевых узлов $\gamma=\left(\gamma_{1}, \ldots, \gamma_{s}\right)$, для которых не существует ненулевого узла $\eta=\left(\eta_{1}, \ldots, \eta_{s}\right)$ из $\Gamma$ с $\left|\eta_{i}\right| \leqslant\left|\gamma_{i}\right|$ при всех $i=1, \ldots, s$ и $\left|\eta_{j}\right|<\left|\gamma_{j}\right|$ хотя бы при одном $i=j$. Следуя Вороному [2], мы будем называть элементы множества $\mathfrak{M}(\Gamma)$ относительными минимумами (см. также [5, гл. Х, §8] по этому поводу).

Хорошо известно, что для двумерных решеток $\mathfrak{N}(\Gamma)=\mathfrak{M}(\Gamma)$, за исключением решеток

$$
\Gamma(u, v)=\left\{\left(m_{1} u+m_{2} u, m_{1} v-m_{2} v\right) \mid m_{1}, m_{2} \in \mathbb{Z}\right\}
$$

с положительными вещественными $u$ и $v$, для которых

$$
\begin{aligned}
\mathfrak{N}(\Gamma(u, v)) & =\{ \pm(2 u, 0), \pm(0,2 v)\}, \\
\mathfrak{M}(\Gamma(u, v)) & =\{ \pm(2 u, 0), \pm(u, v), \pm(u,-v), \pm(0,2 v)\} .
\end{aligned}
$$

Кроме того, для решеток

$$
\Gamma_{\alpha}=\left\{m_{1}(1, \alpha)+m_{2}(0,1) \mid m_{1}, m_{2} \in \mathbb{Z}\right\}
$$

c $\alpha \in(0,1 / 2)$

$$
\mathfrak{N}\left(\Gamma_{\alpha}\right)=\mathfrak{M}\left(\Gamma_{\alpha}\right)=\left\{ \pm\left(Q_{i}, \alpha Q_{i}-P_{i}\right) \mid i=0,1, \ldots\right\}
$$

где $Q_{0}=0, P_{0}=1$, а для $i \geqslant 1$

$$
P_{i} / Q_{i}=\left[0 ; t_{1}, \ldots, t_{i-1}\right]
$$

есть $i$-я подходящая дробь к

$$
\alpha=\left[0 ; t_{1}, \ldots, t_{i}, \ldots\right] .
$$

*Работа выполнена при финансовой поддержке РФФИ, грант №04-01-97000, и INTAS, грант № 03-51-5070. 
Последнее равенство представляет собой разложение числа $\alpha$ в цепную дробь с натуральными $t_{i}$ (неполные частные).

Особо отметим, что конструкция Вороного и Минковского мотивирована классической теоремой Лагранжа о наилучших приближениях с помощью подходящих дробей (см. [6]).

Среди специалистов широко распространено мнение о том, что, начиная с размерности три, описанные выше конструкции приводят к разным множествам. На самом деле это не совсем так. Об этом свидетельствует

Теорема. Для любой $s$-мерной решетки Г имеет место включение $\mathfrak{N}(\Gamma) \subset$ $\mathfrak{M}(\Gamma)$.

ДокАЗАТЕЛЬСтво. Для любого набора $\theta=\left(\theta_{1}, \ldots, \theta_{s}\right)$ из \pm 1 определим решетку

$$
\Gamma^{(\theta)}=\left\{\left(\theta_{1} \gamma_{1}, \ldots, \theta_{s} \gamma_{s}\right) \mid \gamma=\left(\gamma_{1}, \ldots, \gamma_{s}\right) \in \Gamma\right\} .
$$

Из определений немедленно следует, что преобразование

$$
x=\left(x_{1}, \ldots, x_{s}\right) \longmapsto\left(\theta_{1} x_{1}, \ldots, \theta_{s} x_{s}\right)=x^{(\theta)}
$$

переводит $\mathfrak{N}(\Gamma)$ в $\mathfrak{N}\left(\Gamma^{(\theta)}\right)$ и $\mathfrak{M}(\Gamma)$ в $\mathfrak{M}\left(\Gamma^{(\theta)}\right)$. Поэтому утверждение теоремы достаточно доказать для вершин многогранника Клейна $\mathscr{K}_{+}(\Gamma)=\mathscr{K}_{\theta}(\Gamma)$ с $\theta_{1}=$ $\cdots=\theta_{i}=\cdots=\theta_{s}=1$.

Пусть $\gamma-$ произвольная вершина из $\mathscr{K}_{+}(\Gamma)$. Назовем плоскость

$$
\pi(\alpha)=\left\{x \in \mathbb{R}^{s} \mid\langle\alpha, x\rangle=\alpha_{1} x_{1}+\cdots+\alpha_{s} x_{s}=1\right\}
$$

допустимой для $\gamma$, если

1) $\alpha_{i} \geqslant 0$ при всех $i=1, \ldots, s$;

2) $\langle\alpha, \gamma\rangle=1$ и для любого ненулевого узла $\gamma^{\prime}$ решетки $Г$ в угле (1) выполняется неравенство $\left\langle\alpha, \gamma^{\prime}\right\rangle \geqslant 1$.

Равенство $\langle\alpha, \gamma\rangle=1$ означает, что $\gamma$ лежит на плоскости $\pi(\alpha)$. Так как $\gamma-$ вершина многогранника Клейна $\mathscr{K}_{+}(\Gamma)$, то найдутся $s$ допустимых для $\gamma$ плоскостей

$$
\pi\left(\alpha^{(1)}\right), \ldots, \pi\left(\alpha^{(s)}\right)
$$

с линейно независимыми векторами $\alpha^{(1)}, \ldots, \alpha^{(s)}$.

Предположим, что $\gamma$ не есть относительный минимум решетки $Г$. Тогда найдется ненулевой узел $\eta$ решетки $\Gamma$, для которого $\left|\eta_{i}\right| \leqslant\left|\gamma_{i}\right|$ при всех $i$ и $\left|\eta_{j}\right|<\left|\gamma_{j}\right|$ при некотором $i=j$. Заметим, что оба узла $\gamma+\eta$ и $\gamma-\eta$ расположены в угле (1). По условию 2)

$$
1 \leqslant\left\langle\alpha^{(k)}, \gamma \pm \eta\right\rangle=1 \pm\left\langle\alpha^{(k)}, \eta\right\rangle .
$$

Поэтому $\left\langle\alpha^{(k)}, \eta\right\rangle=0$ при всех $k=1, \ldots, s$. Но векторы $\alpha^{(1)}, \ldots, \alpha^{(s)}$ линейно независимы. Следовательно, узел $\eta$ нулевой и мы пришли к противоречию. Теорема полностью доказана. 


\section{ЛитерАТУРА}

1. Klein F. Nachr. Ges. Wiss. Göttingen, No. 3, 357-359 (1895). 2. Вороной Г. Ф. Собрание сочинений в 3-х томах. Т. 1. Изд-во АН УССР, Киев, 1952. 3. Minkowski H. Ann. Sci. École Norm. Sup., Ser. 3, 13, No. 2, 41-60 (1896). 4. Арнольд В. И. Цепные дроби. МЦНМО, М., 2000. 5. Касселс Дж. Введение в геометрию чисел. Мир, М., 1965. 6. Касселс Дж. Введение в теорию диофантовых приближений. ИЛ, М., 1961.

Хабаровское отделение Института прикладной математики Дальневосточного отделения Российской академии наук e-mail: vab@iam.khv.ru
Поступило в редакцию 21 апреля 2004 г. 